\title{
Equivariant extensions of differential forms for non-compact Lie groups
}

\author{
Hugo García-Compeán, Pablo Paniagua, and Bernardo Uribe \\ In celebration of the fiftieth anniversary of the Mathematics Department at the CINVESTAV.
}

\begin{abstract}
Consider a manifold endowed with the action of a Lie group. We study the relation between the cohomology of the Cartan complex and the equivariant cohomology by using the equivariant De Rham complex developed by Getzler, and we show that the cohomology of the Cartan complex lies on the $0-t h$ row of the second page of a spectral sequence converging to the equivariant cohomology. We use this result to generalize a result of Witten on the equivalence of absence of anomalies in gauged WZW actions on compact Lie groups to the existence of equivariant extension of the WZW term, to the case on which the gauge group is the special linear group with real coefficients.
\end{abstract}

\section{Introduction}

In certain situations, geometrical information of manifolds might be encoded in differential forms. In the presence of symmetries of the manifold via the action of a Lie group, the behavior of these differential forms under the group action may lead to a better understanding of the manifold itself. In some well known instances of actions of compact Lie groups, the action is of a particular type whenever the differential form may be extended to an equivariant one in the Cartan model of equivariant cohomology [5]; this is the case for example in Hamiltonian actions on symplectic manifolds [1], in Hamiltonian actions on exact Courant algebroids [4, 6, 12, 14] or in gauged WZW actions which are anomaly free [16] and its generalizations 9, 13].

When the Lie group is not compact, the Cartan complex associated to the action in general does not compute the equivariant cohomology of the manifold, and therefore many of the results that hold for compact Lie groups may not hold for the non-compact case. But since the Cartan model is very well suited for studying the infinitesimal behavior of the differential forms with respect to the action of the

2010 Mathematics Subject Classification. 57R91, 57T10, 81T40, 81T70

Key words and phrases. Equivariant cohomology, gauged WZW action, equivariant extension.

The third author acknowledges and thanks the financial support of the Alexander Von Humboldt Foundation.

(C) 0000 (copyright holder) 
Lie algebra, it would be worthwhile knowing more about the relation between the cohomology of the Cartan complex and the equivariant cohomology. In this paper we study this relation and we obtain some interesting results which in particular permit us generalize the conditions for cancellation of anomalies on gauge WZW actions developed by Witten [16], to the case on which the gauge group is the non-compact group $S L(n, \mathbb{R})$.

The main ingredient of this work is the equivariant De Rham complex developed by Getzler [10] whose cohomology calculates the equivariant cohomology independent whether the group is compact or not. We show that there is an inclusion of complexes of the Cartan complex into the equivariant De Rham one, and therefore we obtain a homomorphism between the cohomology of the Cartan complex to the equivariant De Rham cohomology. This in particular implies that any closed form in the Cartan complex defines an equivariant cohomology class, but note that the converse may not be true. We furthermore show that there is a spectral sequence converging to the equivariant De Rham cohomology such that its $E_{2}^{*, 0}$-term is isomorphic to the cohomology of the Cartan complex, and in this way an explicit relation between the cohomology of the Cartan complex and the equivariant De Rham cohomology is obtained. Now, since the first page of this spectral sequence could be understood in terms of the differential cohomology of a Lie group, we recall its definition and some of its properties and we reconstruct some calculations for the group $S L(n, \mathbb{R})$.

We conclude this work by applying our results to the gauged WZW actions whenever the gauge group is $S L(n, \mathbb{R})$. We recall the result of Witten which claims that the gauged WZW action on compact Lie groups is anomaly free, if and only if the WZW term $\omega=\frac{1}{12 \pi} \operatorname{Tr}\left(g^{-1} d g\right)^{3}$ can be extended to a closed form in the Cartan complex, and we generalize it to the case on which the gauge group is $S L(n, \mathbb{R})$; this is Theorem 4.1. We use this theorem to construct explicit examples where the equations representing the condition of anomalies cancelation hold and where they do not. The physical implications of our work will appear elsewhere.

Acknowledgments The first author was partly supported by the CONACyTMéxico through grant number 128761. The third author would like to acknowledge and to thank the financial support provided by the Alexander Von Humboldt Foundation and also would like to thank the hospitality of Prof. Wolfgang Lück at the Mathematics Institute of the University of Bonn.

\section{Equivariant cohomology}

Let $G$ be a Lie group and $M$ a manifold on which $G$ acts on the left by diffeomorphisms . The $G$-equivariant cohomology of $M$ could be defined as the singular cohomology of the homotopy quotient $E G \times_{G} M$

$$
H_{G}^{*}(M ; \mathbb{Z}):=H^{*}\left(E G \times_{G} M ; \mathbb{Z}\right),
$$

where $E G$ is the universal $G$-principal bundle $G \rightarrow E G \rightarrow B G$.

The previous definition works for any topological group and any continuous action, but sometimes it is convenient to have a De Rham version with differentiable forms for the equivariant cohomology whenever the group is of Lie type and the action is differentiable. When the group $G$ is compact, the Weil and Cartan models provide a framework on which the equivariant cohomology could be obtained via a 
complex whose ingredients are the local action of the Lie algebra $\mathfrak{g}$, and the differentiable forms $\Omega^{\bullet} M$. When the group $G$ is not compact there is a more elaborate model for equivariant cohomology that we will describe in the next section.

2.1. De Rham model of Equivariant cohomology. One way to obtain a De Rham model for equivariant cohomology is through the total complex of the double complex

$$
\Omega^{*}\left(N_{\bullet}(G \ltimes M)\right)
$$

that is obtained after applying the differentiable forms functor to the simplicial space $N_{\bullet}(G \ltimes M)$ which is the nerve of the differentiable groupoid $G \ltimes M$.

By the works of Bott-Shulman-Stasheff [3] and Getzler [10] we know that one way to calculate the cohomology of the total complex of the double complex of differentiable forms

$$
\Omega^{*}\left(N_{\bullet}(G \ltimes M)\right)
$$

is through the differentiable cohomology groups

$$
H^{*}\left(G, S \mathfrak{g}^{*} \otimes \Omega^{\bullet} M\right)
$$

of the group $G$ with values on the differentiable forms of $M$ tensor the symmetric algebra of the dual of the Lie algebra $\mathfrak{g}$.

In [10] Getzler has shown that there is a De Rham theorem for equivariant cohomology showing that there is an isomorphism of rings

$$
H^{*}\left(G, S \mathfrak{g}^{*} \otimes \Omega^{\bullet} M\right) \cong H^{*}\left(E G \times_{G} M ; \mathbb{R}\right)
$$

between the De Rham model for equivariant cohomology and the cohomology of the homotopy quotient.

The De Rham model for equivariant cohomology defined by Getzler is described as follows.

Consider the complex $C^{k}\left(G, S \mathfrak{g}^{*} \otimes \Omega^{\bullet} M\right)$ with elements smooth maps

$$
f\left(g_{1}, \ldots, g_{k} \mid X\right): G^{k} \times \mathfrak{g} \rightarrow \Omega^{\bullet} M,
$$

which vanish if any of the arguments $g_{i}$ equals the identity of $G$. The operators $d$ and $\iota$ are defined by the formulas

$$
\begin{aligned}
& (d f)\left(g_{1}, \ldots, g_{k} \mid X\right)=(-1)^{k} d f\left(g_{1}, \ldots, g_{k} \mid X\right) \quad \text { and } \\
& (\iota f)\left(g_{1}, \ldots, g_{k} \mid X\right)=(-1)^{k} \iota(X) f\left(g_{1}, \ldots, g_{k} \mid X\right),
\end{aligned}
$$

as in the case of the differential in Cartan's model for equivariant cohomology [5, 11]. Recall that the elements in $\mathfrak{g}^{*}$ are defined to have degree 2 , and therefore the operator $\iota$ has degree 1. Denote the generators of the symmetric algebra $S \mathfrak{g}^{*}$ by $\Omega^{a}$ where $a$ runs over a base of $\mathfrak{g}$.

The coboundary $\bar{d}: C^{k} \rightarrow C^{k+1}$ is defined by the formula

$$
\begin{aligned}
(\bar{d} f)\left(g_{0}, \ldots, g_{k} \mid X\right)= & f\left(g_{1}, \ldots, g_{k} \mid X\right)+\sum_{i=1}^{k}(-1)^{i} f\left(g_{0}, \ldots, g_{i-1} g_{i}, \ldots, g_{k} \mid X\right) \\
& +(-1)^{k+1} g_{k} f\left(g_{0}, \ldots, g_{k-1} \mid \operatorname{Ad}\left(g_{k}^{-1}\right) X\right),
\end{aligned}
$$

and the contraction $\bar{\iota}: C^{k} \rightarrow C^{k-1}$ is defined by the formula

$$
(\bar{\iota} f)\left(g_{1}, \ldots, g_{k-1} \mid X\right)=\sum_{i=0}^{k-1}(-1)^{i} \frac{\partial}{\partial t} f\left(g_{1}, \ldots, g_{i}, e^{t X_{i}}, g_{i+1} \ldots, g_{k-1} \mid X\right),
$$


where $X_{i}=\operatorname{Ad}\left(g_{i+1} \ldots g_{k-1}\right) X$.

If the image of the map

$$
f: G^{k} \rightarrow S \mathfrak{g}^{*} \otimes \Omega^{\bullet} M
$$

is a homogeneous polynomial of degree $l$, then the total degree of the map $f$ equals $\operatorname{deg}(f)=k+l$. It follows that the structural maps $d, \iota, \bar{d}$ and $\bar{\iota}$ are degree 1 maps, and the operator

$$
d_{G}=d+\iota+\bar{d}+\bar{\iota}
$$

becomes a degree 1 map that squares to zero.

Definition 2.1. The elements of the complex

$$
\left(C^{*}\left(G, S \mathfrak{g}^{*} \otimes \Omega^{\bullet} M\right), d_{G}\right)
$$

will be called equivariant De Rham forms and its cohomology

$$
H^{*}\left(G, S \mathfrak{g}^{*} \otimes \Omega^{\bullet} M\right)
$$

will be called the equivariant De Rham cohomology.

In [10] it was shown that the complex $\left(C^{*}\left(G, S \mathfrak{g}^{*} \otimes \Omega^{\bullet} M\right), d_{G}\right)$ together with the cup product

$(a \cup b)\left(g_{1}, \ldots, g_{k+l} \mid X\right)=(-1)^{l(|a|-k)} \gamma a\left(g_{1}, \ldots, g_{k} \mid \operatorname{Ad}\left(\gamma^{-1}\right) X\right) b\left(g_{k+1}, \ldots, g_{k+l} \mid X\right)$

for $\gamma=g_{k+1} \ldots g_{k+l}$, becomes a differential graded algebra, and moreover that there is a canonical isomorphism of rings

$$
H^{*}\left(G, S \mathfrak{g}^{*} \otimes \Omega^{\bullet} M\right) \cong H^{*}\left(M \times_{G} E G ; \mathbb{R}\right)
$$

with the cohomology of the homotopy quotient.

2.1.1. Cartan model for equivariant cohomology. The Cartan model for equivariant cohomology is the differential graded algebra

$$
C_{G}^{*}(M):=\left(S \mathfrak{g}^{*} \otimes \Omega^{\bullet} M\right)^{G}
$$

endowed with the differential $d+\iota$. Therefore there is a natural homomorphism of differential graded algebras

$$
i:\left(C_{G}^{*}(M), d+\iota\right) \rightarrow\left(C^{*}\left(G, S \mathfrak{g}^{*} \otimes \Omega^{\bullet} M\right), d_{G}\right)
$$

given by the inclusion

$$
\left(S \mathfrak{g}^{*} \otimes \Omega^{\bullet} M\right)^{G} \subset C^{0}\left(G, S \mathfrak{g}^{*} \otimes \Omega^{\bullet} M\right)
$$

since the restriction of $d_{G}$ to $\left(S \mathfrak{g}^{*} \otimes \Omega^{\bullet} M\right)^{G}$ is precisely $d+\iota$ because the operators $\bar{d}$ and $\bar{\iota}$ act trivially on the invariant elements of $C^{0}\left(G, S \mathfrak{g}^{*} \otimes \Omega^{\bullet} M\right)$.

The induced map on cohomologies

$$
i: H^{*}\left(C_{G}^{*}(M), d+\iota\right) \rightarrow H^{*}\left(G, S \mathfrak{g}^{*} \otimes \Omega^{\bullet} M\right)
$$

is far from being an isomorphism as the case of $M=p t$ and $G=G L(1, \mathbb{R})_{+}=\mathbb{R}_{+}^{*}$ shows.

In this case $C_{G}(M)=S(\mathfrak{g l}(1, \mathbb{R}))=\mathbb{R}[x]$ and $d+\iota=0$, hence $H^{*}\left(C_{G}(M)\right)=\mathbb{R}[x]$ where $|x|=2$. On the other hand $H^{*}\left(G, S \mathfrak{g}^{*}\right)=H^{*}(B G, \mathbb{R})=\mathbb{R}$ since $B G$ is contractible.

Nevertheless, when the Lie group $G$ is compact, the map $i$ induces an isomorphism in cohomology [10]. Now, in order to understand in more detail the relation between the cohomology of the Cartan model and the equivariant cohomology we will introduce a spectral sequence suited for this purpose. 
2.1.2. A spectral sequence for the equivariant De Rham complex. Let us filter the complex $C^{*}\left(G, S \mathfrak{g}^{*} \otimes \Omega^{\bullet} M\right)$ by the degree in $S \mathfrak{g}^{*} \otimes \Omega^{\bullet} M$; namely, if we consider maps

$$
f: G^{k} \rightarrow S \mathfrak{g}^{*} \otimes \Omega^{\bullet} M
$$

with image homogeneous elements of degree $l$, we will denote $\operatorname{deg}_{1}(f)=k$ and $\operatorname{deg}_{2}(f)=l$. Then we can define the filtration

$$
F^{p}:=\left\{f \in C^{*}\left(G, S \mathfrak{g}^{*} \otimes \Omega^{\bullet} M \mid \operatorname{deg}_{2}(f) \geq p\right\}\right.
$$

where $F^{p+1} \subset F^{p}$. We have that the differentials have the following degrees:

$$
\begin{aligned}
\operatorname{deg}_{1}(d) & =0 & \operatorname{deg}_{2}(d) & =1 \\
\operatorname{deg}_{1}(\iota) & =0 & \operatorname{deg}_{2}(\iota) & =1 \\
\operatorname{deg}_{1}(\bar{d}) & =1 & \operatorname{deg}_{2}(\bar{d}) & =0 \\
\operatorname{deg}_{1}(\bar{\iota}) & =-1 & \operatorname{deg}_{2}(\bar{\iota}) & =2
\end{aligned}
$$

and therefore the filtration is compatible with the differentials.

The spectral sequence associated to the filtration $F^{*}$ has for page 0:

$$
E_{0}=\bigoplus_{p} F^{p} / F^{p+1} \cong C^{*}\left(G, S \mathfrak{g}^{*} \otimes \Omega^{\bullet} M\right)
$$

and the 0 -th differential is $d_{0}=\bar{d}$ because the other three differentials raise $d_{e} g_{2}$. Therefore the page 1 is:

$$
E_{1}=H^{*}\left(C^{*}\left(G, S \mathfrak{g}^{*} \otimes \Omega^{\bullet} M\right), \bar{d}\right)
$$

the differentiable cohomology of $G$ with coefficients in the representation $S \mathfrak{g}^{*} \otimes$ $\Omega^{\bullet} M$. The 0 -th row of the first page is precisely the Cartan complex

$$
E_{1}^{*, 0}=H^{0}\left(C^{*}\left(G, S \mathfrak{g}^{*} \otimes \Omega^{\bullet} M\right), \bar{d}\right)=\left(S \mathfrak{g}^{*} \otimes \Omega^{\bullet} M\right)^{G}=C_{G}(M)
$$

and the first differential on this row $d_{1}: E_{1}^{*, 0} \rightarrow E_{1}^{*+1,0}$ becomes precisely the Cartan differential $d+\iota$. Therefore we see that on the second page we get that

$$
E_{2}^{*, 0} \cong H^{*}\left(C_{G}(M), d+\iota\right),
$$

namely that the 0 -th row of the second page is isomorphic to the cohomology of the Cartan complex.

Therefore we conclude that the composition

$$
E_{2}^{*, 0} \rightarrow H^{*}\left(G, S \mathfrak{g}^{*} \otimes \Omega^{\bullet} M\right)
$$

of the surjective homomorphism $E_{2}^{*, 0} \rightarrow E_{\infty}^{*, 0}$ with the inclusion

$$
E_{\infty}^{*, 0} \subset H^{*}\left(G, S \mathfrak{g}^{*} \otimes \Omega^{\bullet} M\right)
$$

is equivalent to the induced map on cohomologies

$$
i: H^{*}\left(C_{G}^{*}(M), d+\iota\right) \rightarrow H^{*}\left(G, S \mathfrak{g}^{*} \otimes \Omega^{\bullet} M\right)
$$

defined previously. 
2.2. Differentiable cohomology of Lie groups. Notice that in De Rham model for equivariant cohomology the operator $\bar{d}$ is defined in a similar fashion as the differential for group cohomology in the case of discrete groups. The cohomology groups defined by the differential $\bar{d}$ are called the differentiable cohomology groups and are defined for any $G$-module $V$; i.e. if $G$ is a Lie group and $V$ is a $G$-module then the differentiable cohomology of $G$ with values in $V$ is the cohomology of the complex $C_{d}^{*}(G, V)$ where $C_{d}^{k}(G, V)$ consists of differentiable maps $f: G^{k} \rightarrow V$ such that $f$ vanishes if any of the arguments $g_{i}$ equals the identity of $G$, and the differential is $\bar{d}$ is defined by

$$
\begin{aligned}
(\bar{d} f)\left(g_{0}, \ldots, g_{k}\right)= & f\left(g_{1}, \ldots, g_{k}\right)+\sum_{i=1}^{k}(-1)^{i} f\left(g_{0}, \ldots, g_{i-1} g_{i}, \ldots, g_{k}\right) \\
& +(-1)^{k+1} g_{k} f\left(g_{0}, \ldots, g_{k-1}\right) .
\end{aligned}
$$

We denote this cohomology by

$$
H_{d}^{*}(G, V):=H^{*}\left(C^{*}(G, V), \bar{d}\right) .
$$

For $V$ in the category of topological $G$-modules, the cohomology groups $H_{d}^{*}(G, V)$ can be seen as the relative derived functor associated to the $G$ invariant submodule $V^{G}$. In particular we have that

$$
H_{d}^{0}(G, V)=V^{G},
$$

and whenever $G$ is compact the functor of taking the $G$-invariant submodule is exact and therefore in that case $H_{d}^{*>0}(G, V)=0$.

REMARK 2.2. The first page of the spectral sequence that was defined in section 2.1 .2

$$
E_{1}=H^{*}\left(C^{*}\left(G, S \mathfrak{g}^{*} \otimes \Omega^{\bullet} M\right), \bar{d}\right)
$$

is precisely the differential cohomology defined above

$$
E_{1}=H_{d}^{*}\left(G, S \mathfrak{g}^{*} \otimes \Omega^{\bullet} M\right)
$$

for the $G$-module $S \mathfrak{g}^{*} \otimes \Omega^{\bullet} M$.

For $V$ a vector space over $\mathbb{R}$, by differentiating the functions from $G^{k}$ to $V$, Van Est [15] proved that for $G$ connected

$$
H_{d}^{*}(G ; V) \cong H^{*}(\mathfrak{g}, \mathfrak{k} ; V)
$$

whenever $K$ is the maximal compact subgroup of $G, \mathfrak{k}$ and $\mathfrak{g}$ are their corresponding Lie algebras, and $H^{*}(\mathfrak{g}, \mathfrak{k} ; V)$ denotes the Lie algebra cohomology defined by Chevalley and Eilenberg in [7].

Whenever the group $G$ has a compact form $G_{u}$, i.e. a compact Lie group whose complexification is isomorphic to the complexification of $G$

$$
G_{\mathbb{C}} \cong\left(G_{u}\right)_{\mathbb{C}}
$$

then we have that

$$
H^{*}(\mathfrak{g}, \mathfrak{k} ; V) \cong H^{*}\left(\mathfrak{g}_{u}, \mathfrak{k} ; V\right) \cong H^{*}\left(G_{u} / K ; V\right),
$$

where the second isomorphism was proved by Chevalley and Eilenberg in 7] for compact Lie groups, and the first isomorphism follows from the isomorphisms

$$
H^{*}(\mathfrak{g}, \mathfrak{k} ; V) \otimes_{\mathbb{R}} \mathbb{C} \cong H^{*}\left(\mathfrak{g}_{\mathbb{C}}, \mathfrak{k}_{\mathbb{C}} ; V_{\mathbb{C}}\right) \cong H^{*}\left(\mathfrak{g}_{u}, \mathfrak{k} ; V\right) \otimes_{\mathbb{R}} \mathbb{C},
$$


which follow from the isomorphism of complex Lie algebras $\mathfrak{g}_{\mathbb{C}} \cong\left(\mathfrak{g}_{u}\right)_{\mathbb{C}}$. In this case we have that the differentiable cohomology of $G$ can be calculated by topological methods, i.e.

$$
H_{d}^{*}(G ; V) \cong H^{*}\left(G_{u} / K ; V\right) .
$$

2.2.1. Example $G=S L(n, \mathbb{R})$. Let us consider the non-compact group $G=$ $S L(n, \mathbb{R})$. In this case we have

$$
\begin{aligned}
\mathfrak{g} & =\mathfrak{s l}(n, \mathbb{R}) & \mathfrak{g}_{\mathbb{C}} & =\mathfrak{s l}(n, \mathbb{C}) \\
\mathfrak{g}_{u} & =\mathfrak{s u}(n) & \mathfrak{k} & =\mathfrak{s o}(n) \\
\mathfrak{k}_{\mathbb{C}} & =\mathfrak{s o}(n, \mathbb{C}) & K & =S O(n) \\
G_{u} & =S U(n) . & &
\end{aligned}
$$

Therefore for $n>2$ we have that

$$
H_{d}^{*}(S L(n, \mathbb{R}), \mathbb{R})=H^{*}(S U(n) / S O(n) ; \mathbb{R})=\Lambda\left[h_{3}, h_{5}, \ldots, h_{\langle n\rangle}\right],
$$

where the degree of $h_{i}$ is $2 i-1$ and $\langle n\rangle$ is the largest odd integer which is less or equal than $n$.

The equivariant De Rham cohomology of this group is $H^{*}\left(G, S \mathfrak{g}^{*}\right)$. The spectral sequence defined in section 22.1 .2 has for first page

$$
E_{1}=H_{d}^{*}\left(G, S \mathfrak{g}^{*}\right) \cong H^{*}\left(\mathfrak{g}, \mathfrak{k} ; S \mathfrak{g}^{*}\right)
$$

and since the algebra $\mathfrak{g}=\mathfrak{s l}(n, \mathbb{R})$ is reductive and $S \mathfrak{g}^{*}$ is a finite dimensional semi-simple $\mathfrak{g}$-module in each degree [2], then we have that

$$
H^{*}\left(\mathfrak{g}, \mathfrak{k} ; S \mathfrak{g}^{*}\right) \cong H^{*}(\mathfrak{g}, \mathfrak{k} ; \mathbb{R}) \otimes\left(S \mathfrak{g}^{*}\right)^{G}
$$

and therefore

$$
E_{1} \cong H_{d}(G ; \mathbb{R}) \otimes\left(S \mathfrak{g}^{*}\right)^{G} .
$$

The ideal of $G$-invariant polynomials is known to be

$$
\left(S \mathfrak{g}^{*}\right)^{G} \cong \mathbb{R}\left[c_{2}, c_{3}, \ldots, c_{n}\right],
$$

where the degree of $c_{i}$ is $2 i$, and so we get that the first page of the spectral sequence converging to $H^{*}\left(G, S \mathfrak{g}^{*}\right)$ is

$$
E_{1} \cong \Lambda\left[h_{3}, h_{5}, \ldots, h_{\langle n\rangle}\right] \otimes \mathbb{R}\left[c_{2}, c_{3}, \ldots, c_{n}\right] .
$$

Since we know that for $n>2$

$$
H^{*}\left(G, S \mathfrak{g}^{*}\right)=H^{*}(B G ; \mathbb{R})=H^{*}(B S O(n) ; \mathbb{R}) \cong \mathbb{R}\left[c_{2}, c_{4}, \ldots c_{2[n / 2]}\right]
$$

is the free algebra on the Pontrjagin classes, we obtain that the $(2 i)$-th differential of the spectral sequence maps the class $h_{i}$ to the class $c_{i}$

$$
h_{i} \stackrel{d_{2 i}}{\longmapsto} c_{i}
$$

In particular we obtain that

$$
\mathbb{R}=H^{4}\left(G, S \mathfrak{g}^{*}\right) \cong E_{\infty}^{4,0} \cong E_{1}^{4,0}=\left(S^{2} \mathfrak{g}^{*}\right)^{G},
$$

and therefore we see that the fourth cohomology group of $B G$ is generated by the $G$-invariant quadratic forms in $\left(S^{2} \mathfrak{g}^{*}\right)^{G}$. 


\section{Equivariant extension of differential forms}

In many instances in geometry, the action of a compact Lie group on a manifold being of a certain kind is equivalent to the existence of an equivariant lift of a specific invariant closed differential form on the Cartan model. Some examples of this phenomenon are the following:

- The action of $G$ on a symplectic manifold $(M, \omega)$ being Hamiltonian is equivalent to the existence of a closed equivariant lift $\tilde{\omega}=\omega+\mu_{a} \Omega^{a} \in$ $C_{G}^{2}(M)$ of the symplectic form; in this case the maps $\mu_{a}: M \rightarrow \mathbb{R}$ may be assembled into a map $\mu: \mathfrak{g} \rightarrow C^{\infty} M, \mu(a):=\mu_{a}$, which becomes the moment map (see [1]).

- The action of $G$ on an exact Courant algebroid $\left(T M \oplus T^{*} M,[,]_{H}\right)$, with Courant-Dorfman bracket twisted by the closed three form $H$, is Hamiltonian provided there exists a closed equivariant lift $\tilde{H}=\omega+\xi_{a} \Omega^{a} \in$ $C_{G}^{3}(M)$ (see [4, 6, 12, 14]).

- Let $\Gamma$ be a connected, simple, simply connected and compact matrix group, i.e. $\Gamma \subset G L(N, \mathbb{R})$, and denote by $\Gamma_{L} \times \Gamma_{R}$ the product of two copies of $\Gamma$ acting on $\Gamma$ on the left by the action

$$
\begin{aligned}
\left(\Gamma_{L} \times \Gamma_{R}\right) \times \Gamma & \rightarrow \Gamma \\
((g, h), k) & \mapsto g k h^{-1} .
\end{aligned}
$$

A subgroup $G \subset \Gamma_{L} \times \Gamma_{R}$ is called an anomaly-free subgroup if there the WZW action could be gauged with respect to the group action given by $G$. In [16] Witten showed that the anomaly-free subgroups are precisely the subgroups $G$ on which the WZW term

$$
\omega=\frac{1}{12 \pi} \operatorname{Tr}\left(g^{-1} d g\right)^{3}
$$

could be lifted to a closed equivariant 3 -form in the Cartan complex $\tilde{\omega}=$ $\omega-\lambda_{a} \Omega^{a} \in C_{G}^{3}(\Gamma)$. (We will elaborate this construction in the next chapter).

The previous examples are not exhaustive, but they give the idea of the general principle. In these cases we have a $G$-invariant closed form $\omega$ and we need to find a closed equivariant lift $\tilde{\omega}$. Note that since the group $G$ is compact, the existence of a closed equivariant lift in the Cartan model is equivalent to the existence of a lift of the cohomology class $[\omega] \in H^{*}(M ; \mathbb{R})$ on the equivariant cohomology $H^{*}\left(E G \times_{G}\right.$ $M ; \mathbb{R})$. Therefore the obstructions of the existence of the equivariant lift could be studied via several methods, for instance, with the use of the Serre spectral sequence associated to the fibration $M \rightarrow E G \times_{G} M \rightarrow B G$, or with the use of the spectral sequence associated to a filtration of the Cartan complex $C_{G}(M)$ given by the degree of $S \mathfrak{g}^{*}$ as it is done in the papers [8, 17].

But what happens in the case that the Lie group $G$ is not compact? We speculate that the situation should be similar, in the sense that the action being of certain kind is equivalent to the existence of a lift on the equivariant De Rham complex defined in section 2.1. This situation has not been explored so far but we believe that the equivariant De Rham complex provides a framework in which actions on non-compact Lie groups could be better understood.

Since in many instances the geometric information is captured by closed forms in the Cartan complex, we would like to study the relation between the cohomology 
of the Cartan complex and the equivariant De Rham cohomology whenever the group is not compact.

3.1. Cartan complex vs. equivariant De Rham complex. Let us consider the diagram of complexes

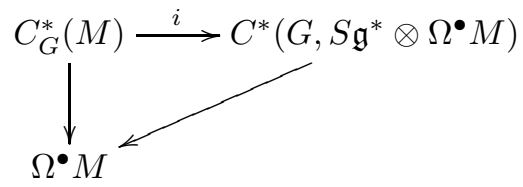

where the horizontal map is the injective map of complexes defined in (2.1) and the vertical maps are the natural forgetful maps. Let us take a closed $G$-invariant form $H$ on $M$ and let us suppose that we can lift this closed form to a closed form in the Cartan model $\bar{H} \in C_{G}(M)$, then equivariant form $i \bar{H}$ becomes a closed lift for $H$ in the complex of equivariant De Rham forms, i.e. $i \bar{H} \in C^{*}\left(G, S \mathfrak{g}^{*} \otimes \Omega^{\bullet} M\right)$. We have then

Lemma 3.1. Take a closed $G$-invariant form $H \in\left(\Omega^{\bullet} M\right)^{G}$. If $H$ can be lifted to a closed form $\bar{H}$ in the Cartan model of the equivariant cohomology, then the form $i(\bar{H})$ is a closed lift for $H$ in $C^{*}\left(G, S \mathfrak{g}^{*} \otimes \Omega^{\bullet} M\right)$, the closed forms of the equivariant De Rahm complex.

Note in particular that Lemma 3.1 implies that if one can extend an invariant closed form to a closed form in the Cartan model for equivariant cohomology, then the cohomology class $[H]$ lies in the image of the canonical forgetful homomorphism

$$
H^{*}\left(G, S \mathfrak{g}^{*} \otimes \Omega^{\bullet} M\right) \rightarrow H^{*}(M),
$$

and therefore the cohomology class $[H]$ could be extended to an equivariant cohomology class in any model for the equivariant cohomology of $M$.

The converse of Lemma 3.1 would say that if one knows that an invariant differentiable form $H$ could be lifted to a closed form in $C^{*}\left(G, S \mathfrak{g}^{*} \otimes \Omega^{\bullet} M\right)$ then a lift could be written as a closed element in the Cartan model. The converse of Lemma 3.1 is indeed true whenever the Lie group $G$ is compact, but for general group actions it does not hold.

From the spectral sequence defined in section $\$ 2.1 .2$ we have seen that in the first page we have

$$
E_{1}^{*, 0} \cong\left(S \mathfrak{g}^{*} \otimes \Omega^{\bullet} M\right)^{G}=C_{G}(M)
$$

with differential $d_{1}: E_{1}^{*, 0} \rightarrow E_{1}^{*+1,0}$ equivalent to $d+\iota$. Therefore on the second page we get that

$$
E_{2}^{*, 0} \cong H^{*}\left(C_{G}(M), d+\iota\right),
$$

namely that the 0 -th row of the second page is isomorphic to the cohomology of the Cartan complex.

Since we have the surjective homomorphism $E_{2}^{*, 0} \rightarrow E_{\infty}^{*, 0}$ we can conclude that

Proposition 3.2. For a closed $G$-invariant form $H \in\left(\Omega^{\bullet} M\right)^{G}$, it can be lifted to a closed form in the Cartan complex if, firstly the cohomology class $[H]$ could be lifted to an equivariant cohomology class in $H^{*}\left(G, S \mathfrak{g}^{*} \otimes \Omega^{\bullet} M\right)$, and secondly, if the lift lies on the subgroup $E_{\infty}^{*, 0} \subset H^{*}\left(G, S \mathfrak{g}^{*} \otimes \Omega^{\bullet} M\right)$. 
The second condition of Proposition 3.2 is more difficult to check than the first one, since it depends explicitly on the equivariant De Rham model for equivariant cohomology; for the first condition any model for the equivariant cohomology works. In certain specific situations, extensions in the Cartan model of closed forms may be obtained, and this is the subject of the next and final chapter.

We note here that a sequence of obstructions for lifting a $G$-invariant form to a closed form in the Cartan complex can be determined, when studying the spectral sequence associated to appropriate filtrations of the Cartan complex, as it is carried out in [8, 17]. Our approach is different since we are interested in using the fact that an extension in the Cartan model can only exist if there is an equivariant extension, i.e. an extension in the homotopy quotient.

\section{Equivariant extensions of the WZW term for $S L(n, \mathbb{R})$ actions}

In the physics literature (see [16] and the references therein) it has been argued that the condition of anomaly cancelation for the gauged WZW action is given by the equation

$$
\operatorname{Tr}\left(T_{a, L} T_{b, L}-T_{a, R} T_{b, R}\right)=0
$$

and this equation is moreover equivalent to the existence of an equivariant extension of the WZW term

$$
\omega=\frac{1}{12 \pi} \operatorname{Tr}\left(g^{-1} d g\right)^{3}
$$

on the Cartan model for equivariant cohomology. In the case that the group that we are gauging is compact, the anomaly cancellation is equivalent to the existence of an equivariant lift of the cohomology class $[\omega]$, and therefore the anomaly cancellation becomes topological and could be checked with topological methods.

In this section we study in detail the case on which the gauge group is $G=$ $S L(n, \mathbb{R})$ (or any subgroup of it) and we show that the anomaly cancelation condition for the WZW action is also topological and only depends on the existence of an equivariant lift of the cohomology class $[\omega]$; in this way we find a large family of $S L(n, \mathbb{R})$ actions with anomaly cancellation.

Let us start by recalling the explanation given by Witten [16] that asserts that the condition for anomaly cancelation is equivalent to the existence of a lift in the Cartan complex of the form $\omega$.

4.1. Gauged WZW actions. Let $\Gamma$ be a connected and simple matrix group, i.e. $\Gamma \subset G L(N, \mathbb{R})$, such that its fundamental group is finite. Denote by $\Gamma_{L} \times \Gamma_{R}$ the product of two copies of $\Gamma$ acting on $\Gamma$ on the left by the action

$$
\begin{aligned}
\left(\Gamma_{L} \times \Gamma_{R}\right) \times \Gamma & \rightarrow \Gamma \\
((g, h), k) & \mapsto g k h^{-1}
\end{aligned}
$$

and consider a subgroup $G \subset \Gamma_{L} \times \Gamma_{R}$ acting on the left on $\Gamma$ by the induced action of $\Gamma_{L} \times \Gamma_{R}$.

The embedding $G \subset \Gamma_{L} \times \Gamma_{R}$ determines a map at the level of Lie algebras which can be written as

$$
a \mapsto\left(T_{a, L}, T_{a, R}\right), \quad a \in \mathfrak{g}
$$

and the canonical vector fields $X_{a}$ on $\Gamma$ generated by the $G$ action could be written as

$$
\left(X_{a}\right)_{g}=T_{a, L} g-g T_{a, R}
$$


for all $g \in \Gamma$.

The matrix 1-forms $g^{-1} d g$ and $d g g^{-1}$ satisfy the equations

$$
\begin{aligned}
\iota_{X_{a}}\left(g^{-1} d g\right) & =g^{-1} T_{a, L} g-T_{a, R}, \\
\iota_{X_{a}}\left(d g g^{-1}\right) & =T_{a, L}-g^{-1} T_{a, R} g, \\
d\left(d g g^{-1}\right)^{2 p+1} & =-\left(d g g^{-1}\right)^{2 p+2}, \\
d\left(g^{-1} d g\right)^{2 p+1} & =\left(g^{-1} d g\right)^{2 p+2}
\end{aligned}
$$

and we can take the differential form

$$
\omega=\frac{1}{12 \pi} \operatorname{Tr}\left(g^{-1} d g\right)^{3},
$$

which defines the WZW action. The form $\omega \in \Omega^{3} \Gamma$ is $\Gamma_{L} \times \Gamma_{R}$ invariant, therefore $\omega \in\left(\Omega^{3} \Gamma\right)^{G}$, it is closed and is a generator of the cohomology group $H^{3}(\Gamma)=\mathbb{R}$.

To find a closed extension of $\omega$ in the Cartan complex we need to find 1-forms $\lambda_{a}$ such that the following equations are satisfied:

$$
\begin{array}{r}
\iota_{X_{a}} \omega-d \lambda_{a}=0, \\
\iota_{X_{a}} \lambda_{b}+\iota_{X_{b}} \lambda_{a}=0, \\
\mathcal{L}_{X_{b}} \lambda_{a}=\lambda_{[b, a]},
\end{array}
$$

where the first two imply that the form $\tilde{\omega}=\omega-\lambda_{a} \Omega^{a}$ is $(d+\iota)$-closed, and the third one implies that $\tilde{\omega}$ is $G$-invariant. Calculating $\iota_{X_{a}} \omega$ we obtain

$$
\begin{aligned}
\iota_{X_{a}} \operatorname{Tr}\left(g^{-1} d g\right)^{3} & =3 \operatorname{Tr}\left(\left(g^{-1} T_{a, L} g-T_{a, R}\right)\left(g^{-1} d g\right)^{2}\right) \\
& =3 \operatorname{Tr}\left(T_{a, L}\left(d g g^{-1}\right)^{2}-T_{a, R}\left(g^{-1} d g\right)^{2}\right) \\
& =d\left[3 \operatorname{Tr}\left(T_{a, L}\left(d g g^{-1}\right)+T_{a, R}\left(g^{-1} d g\right)\right)\right]
\end{aligned}
$$

and therefore we see that we can define

$$
\lambda_{a}=\frac{1}{4 \pi} \operatorname{Tr}\left(T_{a, L}\left(d g g^{-1}\right)+T_{a, R}\left(g^{-1} d g\right)\right)
$$

satisfying the equation $\iota_{X_{a}} \omega-d \lambda_{a}=0$. The fact that $\mathcal{L}_{X_{b}} \lambda_{a}=\lambda_{[b, a]}$ is satisfied, is a tedious but straightforward computation.

Now we compute

$$
\iota_{X_{a}} \lambda_{b}+\iota_{X_{b}} \lambda_{a}=\frac{1}{2 \pi} \operatorname{Tr}\left(T_{a, L} T_{b, L}-T_{a, R} T_{b, R}\right)
$$

noting that for $a, b \in \mathfrak{g}$ the function thus defined become constant, and therefore we have that

$$
\tilde{\omega}=\omega-\lambda_{a} \Omega^{a} \in\left(S \mathfrak{g}^{*} \otimes \Omega^{\bullet} \Gamma\right)^{G}
$$

is a 3 -form in the Cartan complex $C_{G}(\Gamma)$ and its failure to be closed is the quadratic form

$$
(d+\iota) \tilde{\omega} \in\left(S^{2} \mathfrak{g}^{*}\right)^{G},
$$

where the coefficient of $\Omega^{a} \Omega^{b}$ of this quadratic form is precisely

$$
-\frac{1}{2 \pi} \operatorname{Tr}\left(T_{a, L} T_{b, L}-T_{a, R} T_{b, R}\right) .
$$

Since it was known in the literature that the condition for the absence of anomalies was

$$
\operatorname{Tr}\left(T_{a, L} T_{b, L}-T_{a, R} T_{b, R}\right)=0,
$$


Witten concluded that the absence of anomalies was equivalent to the existence of a closed extension of $\omega$ in the Cartan complex.

Whenever the Lie group $G$ is compact, the existence of such an extension is equivalent to the existence of an equivariant extension of the cohomology class $[\omega]$, and this can be checked with the use of the Serre spectral sequence associated to the fibration

$$
\Gamma \rightarrow E G \times_{G} \Gamma \rightarrow B G .
$$

The second page of the spectral sequence becomes

$$
E_{2}^{p, q}=H^{p}\left(B G ; H^{q}(\Gamma ; \mathbb{R})\right) \cong H^{p}(B G ; \mathbb{R}) \otimes H^{q}(\Gamma ; \mathbb{R}),
$$

and since $H^{1}(\Gamma ; \mathbb{R})=H^{2}(\Gamma ; \mathbb{R})=0$, the only non-trivial differential that affects $[\omega] \in E_{2}^{0,3}$ is $d_{4}$ thus defining an element

$$
d_{4}([\omega]) \in E_{2}^{4,0} \cong H^{4}(B G ; \mathbb{R}) ;
$$

this implies that the only obstruction to lift $[\omega]$ to an equivariant class is precisely $d_{4}([\omega])$. Since we assumed that $G$ is compact, we know that

$$
H^{4}(B G ; \mathbb{R}) \cong\left(S^{2} \mathfrak{g}^{*}\right)^{G}
$$

and therefore we must have that

$$
d_{4}([\omega])=(d+\iota) \tilde{\omega},
$$

namely that the two obstructions are the same.

The previous argument permits to find several cases on which there is anomaly cancellation. The simplest of all is the adjoint action of $G$ on itself $\Gamma=G$ since in this case the spectral sequence associated to the fibration $E G \times_{G} G_{a d} \rightarrow B G$ always collapses at the second page, and therefore $d_{4}=0$.

4.2. WZW actions with gauge group $G=S L(n, \mathbb{R})$. In this section we will argue that if the gauge group is $G=S L(n, \mathbb{R})$ then the cancellation of anomalies is topological, and therefore it is equivalent to the existence of an equivariant lift of the cohomology class $[\omega]$.

THEOREM 4.1. Let $\Gamma$ be a connected, simple matrix group with finite fundamental group. Let $G=S L(n, \mathbb{R})$ and consider an action on $\Gamma$ defined by an injection $G \subset \Gamma_{L} \times \Gamma_{R}$. The existence of an equivariant extension on $H^{3}\left(E G \times_{G} \Gamma ; \mathbb{R}\right)$ of the cohomology class $[\omega]$ is equivalent to the existence of a closed lift to the Cartan model of $\omega$.

Proof. We know that if there is an extension $\tilde{\omega}$ in the Cartan model, then the cohomology class $[\tilde{\omega}]$ represents the lift in the cohomology group $H^{3}\left(G, S \mathfrak{g}^{*} \otimes \Omega^{\bullet} \Gamma\right)$. To prove the converse we will make use of the constructions and results of sections 2.2.1, \$3 and $\$ 4.1$.

From the equivariant De Rham theorem we know that $H^{*}(B G ; \mathbb{R}) \cong H\left(G, S \mathfrak{g}^{*}\right)$ and therefore we could take the class $d_{4}([\omega])$, which is the obstruction of extending $[\omega]$ to an equivariant class, to be an element in

$$
d_{4}([\omega]) \in H^{4}\left(G, S \mathfrak{g}^{*}\right) .
$$

We already know that $(d+\iota) \tilde{\omega} \in\left(S^{2} \mathfrak{g}^{*}\right)^{G}$ and its cohomology class in $H^{4}\left(G, S \mathfrak{g}^{*}\right)$ represents the same obstruction for an equivariant lift, i.e.

$$
d_{4}([\omega])=[(d+\iota) \tilde{\omega}] .
$$


In general it may happen that the cohomology class $[(d+\iota) \tilde{\omega}]$ is zero even though the form $(d+\iota) \tilde{\omega}$ is different from zero. But in the particular case of $G=S L(n, \mathbb{R})$ we have already seen in (2.2) that the inclusion map of the Cartan complex into the equivariant De Rham complex

$$
\left(S \mathfrak{g}^{*}\right)^{G} \rightarrow C^{*}\left(G, S \mathfrak{g}^{*}\right)
$$

induces an isomorphism in degree 4

$$
\left(S^{2} \mathfrak{g}^{*}\right)^{G} \stackrel{\cong}{\rightarrow} H^{4}\left(G, S \mathfrak{g}^{*}\right), \quad(d+\iota) \tilde{\omega} \mapsto[(d+\iota) \tilde{\omega}]
$$

and therefore we have that the vanishing of the class $d_{4}([\omega])$ is equivalent to the vanishing of the quadratic form $(d+\iota) \tilde{\omega}$, i.e.

$$
d_{4}([\omega])=0 \text { if and only if }(d+\iota) \tilde{\omega}=0 .
$$

We see that for the case on which the gauge group is $S L(n, \mathbb{R})$, the equations of cancellation of anomalies, namely that for all $a, b \in \mathfrak{s l}(n, \mathbb{R})$

$$
\operatorname{Tr}\left(T_{a, L} T_{b, L}-T_{a, R} T_{b, R}\right)=0,
$$

are equivalent to the existence of an equivariant extension on $H^{3}\left(E G \times_{G} \Gamma ; \mathbb{R}\right)$ of the cohomology class $[\omega]$. Now we are ready to give examples on both the existence and the non existence of equivariant extensions of $\omega$.

\subsection{Examples.}

4.3.1. Adjoint action. Let $G=\Gamma$ and consider the diagonal injection

$$
G \subset \Gamma_{L} \times \Gamma_{R}, \quad g \mapsto(g, g)
$$

which induces the adjoint action of $G$ on $\Gamma=G^{\text {ad }}$. In this case the cohomology of the homotopy quotient $E G \times_{G} G^{\text {ad }}$ is isomorphic to the cohomology of $G$ tensor the cohomology of $B G$ :

$$
H^{*}\left(E G \times_{G} G^{a d} ; \mathbb{R}\right) \cong H^{*}(B G ; \mathbb{R}) \otimes H^{*}(G ; \mathbb{R}) .
$$

This isomorphism can be obtained from the Serre spectral sequence associated to the fibration

$$
G \rightarrow E G \times{ }_{G} G^{\mathrm{ad}} \rightarrow B G
$$

which collapses at level 2 because the classes in $H^{*}(G ; \mathbb{R})$ can be lifted to classes to $H^{*}\left(E G \times{ }_{G} G^{a d} ; \mathbb{R}\right)$ : take a primitive class in $H^{*}(B G ; \mathbb{R})$ (namely a class in $H^{*}(B G ; \mathbb{R})$ which is in the image of a primitive element in $H^{*}(G)$ of one of the differentials of the Serre Spectral Sequence associated to the fibration $G \rightarrow E G \rightarrow$ $B G$ ) pull it back to $S^{1} \times \mathcal{L} B G$ via the evaluation map where $\mathcal{L} B G$ is the space of free loops of $B G$, then integrate over $S^{1}$ and get a class in $H^{*}(\mathcal{L} B G ; \mathbb{R})$ of degree 1 less; this class in $H^{*}(\mathcal{L} B G ; \mathbb{R})$ once restricted to the based loops $\Omega B G \simeq G$ is precisely the class in $H^{*}(G)$ that defined the primitive class in $H^{*}(B G)$ that we started with; finally recall that $\mathcal{L} B G \simeq E G \times{ }_{G} G^{a d}$.

If we take $G=\Gamma=S L(n, \mathbb{R})$, we know by Theorem 4.1 that the existence of an equivariant extension of $[\omega]$ is equivalent to the cancelation of anomalies for this gauged action, and since the class $[\omega]$ can be extended to an equivariant one, we conclude that in this case there is an anomaly cancelation.

In particular, for any subgroup $F \subset S L(n, \mathbb{R})$ acting by the adjoint action on $S L(n, \mathbb{R})$ there is also cancelation of anomalies. 
4.3.2. $G=S L(n, \mathbb{R}) \subset \Gamma_{L}$ for $n>2$. Whenever the action of $G=S L(n, \mathbb{R})$ on $\Gamma$ is obtained by a left action induced by an inclusion $G=S L(n, \mathbb{R}) \subset \Gamma_{L}$, we have that the $G$ action on $\Gamma$ is free and therefore the homotopy quotient $E G \times_{G} \Gamma$ and the quotient $G \backslash \Gamma$ are homotopy equivalent. For $n>2$ the Serre spectral sequence tells us that

$$
d_{4}([\omega])=c_{2} \in H^{4}(B S L(n, \mathbb{R}) ; \mathbb{R})
$$

and therefore the class $[\omega]$ does not extend to an equivariant one. By Theorem 4.1 we know that this implies that there is no cancellation of anomalies. We conclude that for left free actions of the group $S L(n, \mathbb{R})$ for $n>2$ there must exist $a, b \in$ $\mathfrak{s l}(n, \mathbb{R})$ such that

$$
\operatorname{Tr}\left(T_{a, L} T_{b, L}-T_{a, R} T_{b, R}\right) \neq 0 .
$$

4.3.3. $G=S L(2, \mathbb{R}) \subset \Gamma_{L}$. Following the same argument as before, we have that $d_{4}([\omega])=0$ since $H^{4}(B S L(2, \mathbb{R}), \mathbb{R})=0$. Therefore for left free actions of the group $S L(2, \mathbb{R})$ there is anomaly cancelation.

\section{References}

[1] M. F. Atiyah and R. Bott. The moment map and equivariant cohomology. Topology, 23(1):1-28, 1984.

[2] M. Berger et al. Séminaire "Sophus Lie" de l'Ecole Normale Supérieure, 1954/1955. Théorie des algèbres de Lie. Topologie des groupes de Lie. Secrétariat mathématique, 11 rue Pierre Curie, Paris, 1955.

[3] R. Bott, H. Shulman, and J. Stasheff. On the de Rham theory of certain classifying spaces. Adv. Math., 20(1):43-56, 1976.

[4] H. Bursztyn, G. R. Cavalcanti and M. Gualtieri. Reduction of Courant algebroids and generalized complex structures. Adv. Math., 211(2):726-765, 2007.

[5] H. Cartan. La transgression dans un groupe de Lie et dans un espace fibré principal. In Colloque de topologie (espaces fibrés), Bruxelles, 1950, pages 57-71. Georges Thone, Liège, 1951.

[6] A. Caviedes, S. Hu and B. Uribe. Chern-Weil homomorphism in twisted equivariant cohomology. Differential Geom. Appl., 28(1):65-80, 2010.

[7] C. Chevalley and S. Eilenberg. Cohomology theory of Lie groups and Lie algebras. Trans. Amer. Math. Soc., 63:85-124, 1948.

[8] J. M. Figueroa-O'Farrill and S. Stanciu. Gauged Wess-Zumino terms and equivariant cohomology. Phys. Lett. B, 341(2):153-159, 1994.

[9] H. García-Compeán and P. Paniagua. Gauged WZW models via equivariant cohomology. Mod. Phys. Lett. A, 26(18):1343-1352, 2011.

[10] E. Getzler. The equivariant Chern character for non-compact Lie groups. Adv. Math., 109(1):88-107, 1994.

[11] V. W. Guillemin and S. Sternberg. Supersymmetry and equivariant de Rham theory. Mathematics Past and Present. Springer-Verlag, Berlin, 1999.

[12] S. Hu. Reduction and duality in generalized geometry. J. Symplectic Geom., 5(4):439-473, 2007.

[13] P. Paniagua. Anomalías, Teoría de Wess-Zumino-Witten y cohomología equivariante. PhD thesis, CINVESTAV, México, 2010.

[14] B. Uribe. Group actions on dg-manifolds and exact courant algebroids. Comm. Math. Phys., 318(1):35-67, 2013. 
[15] W. T. Van Est. Group cohomology and Lie algebra cohomology in Lie groups. I, II. Nederl. Akad. Wetensch. Proc. Ser. A. 56 = Indagationes Math., 15:484492, 493-504, 1953.

[16] E. Witten. On holomorphic factorization of WZW and coset models. Comm. Math. Phys., 144(1):189-212, 1992.

[17] S. Wu. Cohomological obstructions to the equivariant extension of closed invariant forms. J. Geom. Phys., 10(4):381-392, 1993.

Departamento de Física, Centro de Investigación y de Estudios Avanzados, Av. I.P.N 2508, Zacatenco, Delegación Gustavo A. Madero, C.P. 07360, , México D.F., México

E-mail address: compean@fis.cinvestav.mx

Departamento de Matemáticas, Escuela Superior de Física y Matemáticas del Instituto Politécnico Nacional, Unidad Adolfo López Mateos, Edificio 9, 07738, México D.F., MÉXICO

E-mail address: ppaniagua@esfm.ipn.mx

Departamento de Matemáticas, Universidad de los Andes, Carrera 1 N. 18A - 10, Bogotá, COLOMBIA

E-mail address: buribe@uniandes.edu.co 